REVIEW ARTICLE

\title{
Probiotic foods: Benefits to the cereal based Sri Lankan diet
}

\author{
Anjani M. Karunaratne \\ Department of Botany, Faculty of Science, University of Peradeniya, Peradeniya, Sri Lanka
}

Received:13/10/2017; Accepted:25/12/2017

\begin{abstract}
The Sri Lankan diet of which the staple is rice, has unacceptable levels of antinutritional substances, particularly phytic acid, which is associated with fibre, a dietary component originating from plant sources of food. Low anthropometric indicators among preschool children suggestive of undernutrition have remained static over several years. Some microbes that ferment food are known to produce phytase that metabolize phytic acid in addition to providing other benefits. Some of these microbes in fermented foods serve as probiotics, which help maintain homeostasis within the gut microbiota, thus providing a wide range of health benefits. At present, it is a worldwide trend to incorporate probiotics to the daily diet in fermented foods, while a market for novel probiotic foods is catching on. In the gut, probiotics can thrive on indigestible carbohydrates (fibre) serving as prebiotics. Therefore, designing synbiotic foods having both probiotics and prebiotics with enhanced benefits is also becoming popular globally. This review evaluates the feasibility of introducing a wide range of fermented foods to the Sri Lankan diet. Such a move is envisaged to diversify the local diet thus helping to alleviate certain nutritional shortcomings. This can subsequently help in the introduction of specific probiotics with beneficial effects.
\end{abstract}

Keywords: gut microbiota, paraprobiotics, malnutrition, food fermentation, non-communicable diseases, preschool children.

\section{INTRODUCTION}

In any part of Sri Lanka, the staple diet composes mainly of rice (about $300-400 \mathrm{~g}$ ) (FBDG, 2011) with some vegetables and typically a small portion (about $15 \mathrm{~g}$ ) of meat or fish (Jayawardena et al., 2012), or a chicken egg (whole or half). While the intake of specific quantities from a variety of food is recommended for Sri Lankans (FBDG, 2011), the bulk of the food consumed by the average Sri Lankan can be considered as composed of rice with a few vegetables (Jayawardena et al., 2012). Tudawe and Wikramanayake (2000) fittingly document the diet of the rural populations in Sri Lanka as being high in cereals with low intake of foods of animal origin. In fact this description seems to cover the typical diet of the average Sri Lankan and may not be restricted to rural populations alone. Although, globally it is an accepted fact that a well planned vegetarian diet is healthy (Agnoli et al., 2017), it is proven beyond doubt that a primarily vegetarian diet unless properly planned, could result in deficiencies in terms of protein and micronutrients. A well-balanced Sri Lankan diet composed of rice, spicy vegetable curries and protein sources (pulses or food of animal origin) at recommended portion sizes (FBDG, 2011), is enriched with dietary fibre and antioxidants and hence, has the potential of being a healthy meal. However, as consumed by the masses, the diet lacks both balance and variety (Jayawardena et al., 2012) which seems to have severe repercussions on the nutritional status and health. In addition to several studies reporting the prevalence of undernutrition among children (as discussed later), a national nutritional survey (Jayatissa et al., 2012) shows the prevalence of malnutrition (both undernutrition and overnutrition) in the adult population as well.

Even the diets of children do not differ greatly from that of adults. The level of the antinutritional substance phytic acid is unacceptably high in certain combinations of meals of preschool children (3- 5 year olds) (Karunaratne et al., 2008). Multiple micronutrient deficiencies are recorded from the southern parts of Sri Lanka in a study carried out on school children (12-15 year olds) in spite of the subjects being clinically healthy (Hettiarachchi et al., 2006). Also, a more recent study carried out in the south of the country on younger children (18-24 months), a high prevalence of undernutrition based on anthropometric parameters is reported with the levels of wasting and underweight showing an increase with increasing age (Ubeysekara et al., 2015). Similarly, on a study on rural preschool children (3- 5 year olds) in central Sri Lanka, low anthropometric parameters are reported, indicating undernutrition with inability to discern specific limiting nutrients (Karunaratne, 2007). In fact, with single micronutrient supplementations not having yielded satisfactory results the existence of multiple micronutrient deficiencies in developing countries is evident (Hettiarachchi et al., 2006). A national micronutrient survey reports anthropometric indicators among 7306 preschool children (6-59 months) with stunting, wasting and underweight percentages at $13.1 \%$, $19.6 \%$, and $23.5 \%$ respectively (Jayatissa et al., 2012). The above studies indicate that undernutrition is a major public health problem in Sri Lanka affecting children. With such a long chronological span of studies consistently reporting undernutrition in specific regions, the existence of hidden hunger (that indicates multiple micronutrient deficiencies with no overt symptoms) is apparent. Therefore, the need 
to find solutions to combat this situation is felt deeply. Unfortunately, even Thriposha which is a nutritional supplement containing cereals and pulses, supplied as a remedy to such groups has high levels of phytic acid (Karunaratne, 2007). Improving the inherent quality of the Sri Lankan diet, which has large quantities of dietary fibre associated with the antinutrient phytic acid (Karunaratne, et al., 2008), would be an important step in combating malnutrition.

Ironically dietary fibre has shown to influence positively on the gut microflora, and adding probiotics to the daily diet can be expected to help in reducing antinutritional effects, in addition to improving the micronutrient intake. As the practice of food fermentation has helped overcome hidden hunger (Kavitake et al., 2018) including a variety of fermented foods to the local diet seems to be a sensible decision. A FAO/WHO (2001) report defines the term probiotic as 'for life' and the term is currently used to name bacteria associated with beneficial effects for humans and animals. The most popular method of introducing probiotics to a food is by using them to ferment a food substrate (Kavitake et al., 2018). Probiotics are much researched microbes that are included in an important group of functional foods. The term functional food itself has undergone many definitions over recent years, but all of them acknowledge their health promoting effects (Martirosyan and Singh, 2015). Probiotics have many prophylactic and therapeutic effects through the influence they have on the gut flora. Among the roles they play are to extract nutrients and energy from food consumed, protect against enteropathogens, and contribute to normal immune function (Lozupone et al., 2012). In fact traditional fermented food sources and the knowledge of their health promoting effects preserved in groups of populations where information has been passed from generation to generation has led to the discovery of this group of functional foods.

Dietary diversification is envisaged as being helpful in improving the nutritional quality of the Sri Lankan diet (Karunaratne, 2007; Jayawardena et al., 2012). Among methods of reducing the antinutritional substance, phytic acid in high fibre vegetarian diets are to incorporate phytase producing foods to the daily diet. In many vegetarian diets world wide, microbial phytase (originating from bacteria that participate in the fermentation of foods) and indigenous phytase (originating from sprouting of seeds) are exploited for this purpose. In fact, the addition of fermented foods is recommended internationally, to improve the nutritional attributes of vegetarian diets of developed countries (Agnoli et al., 2017). Referring to the food as consumed in developing countries Gibson and Ferguson (1998) record the importance of incorporation of fermented food sources which probably could compensate for the primarily vegetarian diet rich in antinutrients. Generally, it is acknowledged that the fermentation process replenishes the food substrate with vitamins and improves protein availability besides reducing antinutritional content (Sahlin, 1999). In vitro experiments and studies on humans have documented the capacity of some probiotic strains to synthesize vitamin $\mathrm{K}$, folic Acid (vitamin $\mathrm{B}_{9}$ ), vitamin $\mathrm{B}_{2}$, and $\mathrm{B}_{12}$ (Eck and Friel, 2013). Therefore, including fermented foods in vegetarian diets, will help in overcoming vitamin deficiencies, specially vitamin $\mathrm{B}_{12}$ being the limiting micronutrient (Agnoli et al., 2017) provided the right microorganisms are used in their fermentation.

With the unraveling of the beneficial roles of probiotics there is a worldwide trend to look for probiotic strains and to introduce them to the daily diet, which involves sharing information from different cultures and devising innovative methods. Internationally, fermented milk and dairy products such as curds and yoghurts in solid and liquid forms as well as a variety of fermented cheese have been popular. The diversity of traditional fermented products in India extends to many plant based products like seeds, vegetables, and fruits (Marshall and Mejia, 2011; Blandino et al., 2003; Sahlin, 1999). However such a diversity of traditional fermented foods is not restricted to South Asian countries. A well known global example is cabbage fermentation; originating from Germany is fermented cabbage 'sauerkraut', and in the far Eastern Countries like Korea, its 'kimchi'. The recipe of 'kimchi' differs from that of 'sauerkraut' only by making it spicy. Also a traditional fermented non-alcoholic beverage is 'beet kvass' which originates from Russia. In addition, in the Far Eastern countries many vegetables such as cucumbers and turnips are fermented and served as pickles.

Although traditional preservation methods such as food fermentation probably developed initially only to increase shelf-life of perishable produce, with the advancement and sharing of knowledge, their nutritional and health effects seem to have captured the attention of nutritionists worldwide. Currently, the diverse health promoting roles of specific probiotics which can be incorporated to traditional fermented foods are well documented. This review evaluates the feasibility of introducing a wide range of fermented plant based foods with an attempt to diversify the local diet, so that it will improve the nutritional status. Such an initial move in turn, can help in the introduction of specific probiotics with health beneficial effects to the daily diet.

\section{Balancing the dual role of microbes in health and disease}

Microbes have been seen by the laymen as a cause of human misery through the surfeit of diseases they inflict. This may be the reason for the delay in unveiling their silent role in the wellbeing of man and animals. The purpose of their existence in excessive numbers within and on the healthy body and the colossal role they play in maintaining good health seems to have got obscured due to our preoccupation on microbial pathogens.

Currently, there is much interest on the microbes that inhabit the human body, specifically those in the gut (gut microbiota). It is recorded that not only man, but all mammals have evolved with their commensal partners (microbes), and adaptive co-evolution has formed an inextricable bond between the microbiota and their host (Ventura et al., 2012). Probiotics are of specific importance for enriching the gut microbiota which in turn provides 
health benefits to the host. The ability of probiotics to competitively exclude pathogenic microbes has been documented (Chen et al., 2007; Chervonsky, 2012). Therefore, according to currently accepted knowledge, any food we take on a daily basis which has sufficient viable probiotics, is believed to help in the modulation of the gut microbiota, the term viable being the key word here, until we take up this point again.

Nevertheless, pathogens can be rampant in careless food handling situations. The fresh fruits and vegetables that we consume may be exposed to pathogens, in their cultivating phase or during careless postharvest handling and storage. The tropical temperatures and faulty and ineffective cold chain facilities in handling fresh produce are conducive factors for pathogen establishment. Rizzo et al. (2013) describe how gene exchanges could occur among unrelated organisms, taking microbial niches of waste water treatment plants as an example. Therefore when pathogens lurk in considerable numbers in the food environments, it can be assumed that such microbial niches may be conducive for horizontal gene transfers resulting in dangerous outcomes.

Focusing on the health promoting role of fermented foods, to confer their benefits the widely held belief is that probiotics in food must survive its transit from the mouth to the colon. Being on that stand, the FAO/WHO (2001) defines probiotics as live microorganisms which when administered in adequate numbers, confer health benefits on the host. The numbers of probiotic cells present in a processed food have been worked out. For instance, when populations of $10^{6}-10^{7} \mathrm{CFU} / \mathrm{g}$ in the final product are established, a daily consumption of $100 \mathrm{~g}$ or $100 \mathrm{ml}$ of the food would provide probiotics equivalent to $10^{8}-10^{9} \mathrm{CFU}$ (Coman et al., 2012).

Often traditional fermented foods do not contain a well defined set of microorganisms and such foods are fermented by a mixed culture of microorganisms by spontaneous fermentation (Ray et al., 2016). In them, the conditions conducive for the growth of microorganisms are provided during their preparation which leads to the establishment of a complex ecosystem (Demarigny, 2012). Therefore, it is not possible to assume that all microorganisms in a traditionally fermented food have probiotic potential. Instead, they may compose of a mixture of commensals, probiotics, and perhaps a few pathogenic organisms too. In such an undefined fermenting environment, meeting requirements of probiotic cell numbers to be consumed is not easy without compromising on food safety. When handling situations are not up to the mark, this task may face many obstacles. However, as discussed later in this review, even heat-killed probiotics have shown beneficial effects.

\section{Role of Prebiotics}

The term prebiotic was first introduced by Gibson and Roberfroid (1995) and was defined as "a nondigestible food ingredient that beneficially affects the host by selectively stimulating the growth and/or activity of one or a limited number of bacteria in the colon, and thus improves host health". While sugars such as lactose in milk support the growth of probiotics they are also capable of breaking complex polysaccharides that the human enzymes cannot breakdown. Food fibre mainly composed of polysaccharides, is considered as serving as prebiotics. Fibre from whole grains has captured interest of researchers for a multitude of health benefits (Phillips, 2013) and a recent review by Gong et al. (2018), presents the possible effects of cereal originated dietary fiber on the composition and metabolic activity of the intestinal microbiota. Prebiotics cannot be broken down by human enzymes, but the beta linkages of their chemical structures are broken down by probiotics. Normally, the responses of different bacteria to carbohydrates are usually related to their abilities to encode corresponding enzymes for the utilization of different carbohydrates (Gong et al., 2018).

Among prebiotics there are many health promoting phytochemicals. Oligosaccharides are a much sort after group of prebiotics that are abundantly present in pulses. Before their role as prebiotics were discovered, oligosaccharides were considered as substances that caused annoyance as they are not digested by human enzymes and caused flatulence, causing much discomfort and embarrassment for the consumer. Some examples of oligosaccharide based prebiotics are inulin and oligofructose (produced from inulin), and fructooligosaccharides synthetically produced from sucrose, as well as galactosecontaining and xylose-containing oligosaccharides (Hutkins et al., 2016). In fact, inulin and oligofructose are the most studied prebiotics, and inulin-type prebiotics that are commercially available are those that have been extracted from food or synthesized from a more fundamental molecule (typically sucrose), and both in vitro and in vivo studies have confirmed their selective stimulation of beneficial flora (Gong et al., 2018).

Fruits and vegetables are also rich in fibre thus qualifying as prebiotic sources. Pectins present in the middle lamella of plant cells and therefore found abundantly in plant based foods are also considered as prebiotics (Gibson and Roberfroid, 1995; Ray et al., 2016; Hutkins et al., 2016). Pectins are known to have many health beneficial roles such as reduction of serum low density cholesterol, and thereby decreasing the risk of cardiovascular disease by the binding of bile salt micelles thus inhibiting their re-absorption (Morris et al., 2013). Resistant starch present in partially ripe tropical fruits such as bananas, hitherto appreciated for the role they serve as food fibre is also considered as serving as prebiotics (Thomas et al., 2011; Hutkins et al., 2016; Ray et al., 2016; Gong et al., 2018). Fructans found abundantly in plants as storage carbohydrates are also one of the oldest known prebiotics (Gibson and Ferguson, 1998; Gong et al., 2018). The interest on fructans has grown over the recent years because in addition to being a much sought after prebiotic source, this group of compounds are known to play protective roles in intact plants subjected to stress (Franco-Robles and López, 2015). It is reported that fructans and other prebiotics may suppress pathogen growth within the gut, to improve overall health. Therefore, fructan itself is considered as an excellent functional food 
and novel synbiotic foods (a combination of probiotics and prebiotics) are being experimented with this prebiotic from natural sources (Shalini et al., 2017). Franco-Robles and López (2015) have tabulated the prebiotic effects of fructans in in vitro and in vivo studies and also on its influence on the immune function in healthy animal and human models.

Additionally prebiotics have many functional roles to play in the food thus adding to its appeal. Such supplementary benefits of those substances that qualify as prebiotics in food applications are many; oligosaccharides have been increasingly used by the food industry (beverages, sweets) for modifying viscosity, emulsification capacity, gel formation and enhancing colour (Saad et al., 2013); pectins being excellent gelling agents and emulsifiers, have many food applications in thickening beverages and in desserts. As probiotics thrive on prebiotics, the choice of prebiotics also matter for providing optimal health benefits. Therefore ideally, the choice of prebiotics has to be based on their role in the formulation of the food in question, in addition to the probiotics promoted by them. The knowledge on their ideal combinations for optimal results however, is still to be investigated.

As discussed before, when plant based foods serve as carriers of probiotics there is the added advantage of delivering prebiotics with them, so that the entire food complex becomes a synbiotic 'package'. Fruit, vegetables, cereals and legume products represent promising carriers for probiotic bacteria with good nutraceutical components (Panghal et al., 2018). Gong et al (2018) referring to whole cereal grains explain that they exert their beneficial effects on the health of the host by delivering prebiotics to modulate gut microbiota composition, richness and activities to maintain homeostasis (correct ecological imbalances), and also provide substrates to be metabolized into functional microbiota metabolites. Such events helping to combat non-communicable diseases (NCDs) can originate from the supply of fibre originating from other plant based sources as well, as discussed in the present review.

With this array of food applications and health promoting roles of prebiotics, to harness the benefits of both probiotics and prebiotics, hybrid food products made by combining dairy and fruit drinks have been introduced offering not only healthier but also more convenient and more flavourful products (Martins et al., 2013). With a need for advancement of technology in the introduction of probiotics, specific prebiotics are being experimented as coatings used for microencapsulation of probiotics (Kavitake et al., 2018). In such microencapsulations the prebiotic used has shown to promote growth of the encapsuled probiotics once released to the gut environment (Shori, 2017). Among some prebiotics that have been tried out for encapsulation are inulin and oligofructose (Tripathi and Giri, 2014).

\section{Evolution of probiotic foods at a global level}

Fermented foods are known to be fermented by both functional and non-functional microorganisms that exist as native microflora primarily in raw plant materials and such microbes can alter the biochemical constituents of raw materials, thereby improving the flavor, digestibility and aroma while imparting nutritional and pharmacological values (Kavitake et al., 2018). It appears that the health promoting effects linked to some traditional fermented foods have created the initial impetus for an indepth investigation on probiotics. An overview of the consumption of traditional fermented foods in specific countries is given by Demarigny (2012) while emphasizing the need to adhere to hygienic protocols to prevent the establishment of undesirable organisms. The concept of health promoting probiotics was probably introduced by the Russian Nobel laureate Elie Metchnikoff (1845-1916) when he hypothesized that consuming fermented milk, made Bulgarians live longer because of the activity of lactic acid bacteria-LAB (Metchnikoff, 1908). Dairy products, specifically different types of yoghurts and cheeses have been the most popular foods over the years, to deliver probiotics (Granato et al., 2010 a and b, Asemi et al., 2013; McFarland, 2015).

Currently, the focus has turned to non-dairy fermented foods. Panghal et al., (2018) report that the first probiotic food free from milk or milk constituents was formulated and manufactured in 1994 by Skane Dairy with brand name ProViva in Sweden. As mentioned earlier, some countries use vegetables (Martin et al., 2013; Swain et al., 2014) and seeds (Sahlin, 1999; Blandino et al., 2003; Marshall and Mejia, 2011; Tamang, 2015; Patra et al., 2016) to prepare traditionally fermented foods. At present there is much interest on such foods, as fruits, vegetables, and seeds by themselves are considered healthy options of food, and using such substrates for fermentation is expected to improve the beneficial effects of the final product. Therefore fruits and vegetables have turned out to be new food environments to introduce probiotics with several new products being introduced in the $21^{\text {st }}$ century (Granato et al., 2010a; Martins et al., 2013; Panghal et al., 2018).

This diversification to non-dairy options could be assumed to have occurred not only for the want of variety for palatability but also for practical reasons. For instance, Granato et al. (2010a) record that in countries where the use of dairy products is limited due to reasons such as allergies and lactose intolerance, nondairy probiotic foods have been used. Also there are specific groups of individuals, such as those affected by different types of NCDs, such as dyslipidemia and atherosclerosis thus requiring dietary restrictions. Also, with the increasing vegetarian consumers in both developed and developing countries, there is a high demand for plant based probiotic products (Panghal et al., 2018).

Having identified the health promoting effects of viable probiotic cells per se, currently food technologists are focusing on food products that can only deliver viable probiotics in appropriate numbers to the consumer, without going through the process of fermentation. As a result, according to numerous publications, the delivery of probiotics is shifting towards looking at food substrates that could harbour adequate numbers of viable cells without promoting their growth within the food matrix, thus 
keeping the ingredients of the food intact. Non-alcoholic beverages such as fruit/vegetable drinks and soymilk (soya milk as referred to, in Sri Lanka) serve as carriers of specific probiotics (Granato et al., 2010a). Successes are also reported with regard to ice-cream, frozen desserts, and even baby foods (Tripathi and Giri, 2014).

Although there are certain disadvantages with such alternative preparations, as discussed below, the major advantage is that it provides leverage to work with any type of food. Unlike in traditionally fermented foods, specific probiotic strains of interest could be incorporated to the food in question, while preserving its original organoleptic properties. Therefore, the adaptation of modern technologies to the food industry, in the form of encapsulation and cell protectants (Tripathi and Giri, 2014) help probiotics remain viable without affecting the food matrix. With this widening of resources, an increasing number of foods has been and continues to be experimented with as probiotic carriers (Granato et al., 2010a; Permpoonpattana et al., 2012).

\section{Global interest and diversity of fermented food - Where does Sri Lanka stand?}

New findings continue to get added to the scientific literature on various aspects dealing with the health benefits of probiotics at an exponential rate; According to Taverniti and Guglielmetti (2011), a net search yielded about 7500 scientific references of which more than $20 \%$ appeared in 2010. Later, McFarland (2015) noted that in the year 2000 the number of papers on probiotics was from 176 per year and within 2000 to 2014 this number went up to 1476 per year. These figures testify for the growing interest on the international scenario on probiotic foods.

In spite of such a trend internationally, in Sri Lanka fermented foods that can provide viable probiotics have been mainly restricted to snacks and desserts in the form of curd and yoghurt, the latter was introduced to the Sri Lankan consumer in the late 1960s (personal experience of author). Surprisingly, fermented foods with viable organisms in them don't appear to have been considered an important group of food in ancient Sri Lanka. While reviews have appeared of fermented foods in different countries throughout the world (Kavitake et al., 2018), in Asian countries such as India (Tamang, 2015; Ray et al., 2016), Korea (Patra et al., 2016) and Vietnam (La Anh, 2015) and of fermented fruits and vegetables of Asia in general (Swain et al., 2014; Tamang, 2015) some inclusive of Africa (Demarigny, 2012; Panghal et al., 2018), in Sri Lanka there is not much information available on such traditional indigenous fermented foods. Comparing with our immediate neighbor, India, specially in the southern parts, rice is consumed with a few curries as done in Sri Lanka. While there is a diversity of fermented plant based foods, the consumption of fermented dairy products is an integral part of their daily diet, and many households make their own curd at home on a daily basis. Considering the fact that a large proportion of the population in India is vegetarian, this habit seems to be a way to improve the nutritive value of food consumed daily. Contrarily in Sri Lanka, milk consumption of preschool children (3-5 year olds) is inadequate, for which the reasons given are, nasal blockage caused by phlegm when fresh milk is consumed, and powdered milk is not affordable (Karunaratne, 2007). Additionally, the same study noted that fermented food intake of children was insignificant. Perhaps, being a small tropical country with varied climatic zones, preserving food was not a priority for our ancestors as fresh food has been available all year round.

Unrefined plant based foods such as whole grain seeds, and fresh fruits and vegetables, which are rich in indigestible carbohydrates serve as prebiotics (Gibson, and Roberfroid, 1995; Hutkins et al., 2016). Therefore, the natural sources of prebiotics are indigestible components of plant based foods. The role of prebiotics is to basically support the growth of probiotics within the colon, as they pass through the alimentary canal intact, without being affected by the conditions or the enzymes that they are exposed to, until they enter the gut environment. While the beneficial effects provided by probiotics and prebiotics are well established, the recent worldwide trend is to manufacture novel synbiotic foods. In fact many patents have been obtained for novel probiotic and synbiotic foods specially where the commodity of delivery is fruit (Sun-Waterhouse, 2011). If probiotics are incorporated to the already prebiotic rich food environment of the Sri Lankan diet, such a move may have beneficial effects to the consumer. However, one major challenge with regard to such novel foods is consumer acceptability. According to Panghal et al. (2018) blackcurrant probiotic juice is more acceptable than the conventional blackcurrant juice, but orange probiotic juice is less acceptable than the conventional orange juice. With proper guidelines and technology, using already familiar plant based food ingredients which will be more receptive to the local consumer, developing a local probiotic food market has a great potential

Non-viable cells serving as probiotics in cooked fermented foods

In spite of the fact that uncooked fermented foods are not popular in the local diet, some of the Sri Lankan foods consumed frequently as main meals are fermented, but eventually cooked before consumption. For instance, 'hoppers' (pancakes made in a wok) made of a dough of fermented rice flour, and Indian type fermented staple foods such as 'thosai' (pancake) and 'idli' (steamed cake) made of a dough of fermented black gram (Vigna mungo) are popular breakfast or dinner choices in Sri Lanka.

Of a number of cereal and pulse based indigenous fermented cooked products reported from other countries, many of the ingredients used in such products are commonly used locally. For instance, 'dhokla' is a steamed cake made of a fermented dough of rice or wheat flour and Benghal gram (Kadala in Sinhala, Kadalai in Tamil) (Cicer arietinum) used as a breakfast food in Northern parts of India (Blandino et al., 2003). Another rice based probiotic functional product called 'ricera' is mentioned by Panghal et al. (2018). Also, 'com-me' is a fermented appetizer made of cooked rice popular countrywide in Vietnam (La Anh, 2015). Some fermented preparations of soya bean are cooked as curries in several Asian countries (Tamang, 2015). In Korea 'gochujang' is a fermented chillie-paste 
(Patra et al., 2016).

In such cooked products, the microbial cells involved in the fermentation, are expected to be killed by the cooking temperatures. In spite of the established fact that viable probiotic organisms should reach the gut alive to get their therapeutic benefits, some studies as discussed below have shown that even non-living probiotics may be just as effective. According to the findings from in vitro cell-culture studies and in vivo animal studies many of the effects obtained from viable cells of probiotics are also obtained from populations of dead cells, and therefore, Taverniti and Guglielmetti (2011) proposed the term paraprobiotic to indicate the use of non living probiotic cells. Lately, such studies have been even extended to human subjects (Shinkai et al., 2013; Cerbo et al., 2015). For instance, Shinkai et al. (2013), in a randomized control trial demonstrated that oral intake of a heat-killed strain of Lactobacillus pentosus given as a tablet, dose-dependently reduced the incidence of the common cold in elderly adults leading to the postulation whether only the presence of cell components of probiotics in the gut is sufficient to cause some of the beneficial effects, in the absence of live cells. Sang et al., (2015) demonstrated that a therapeutic mixture known as vsl\#3 made of a probiotic cell combination had identical anti-inflammatory effects whether they were administered as viable cells or heat-killed cells to rats. Additionally, using animal and human subjects it has been shown that a strain of heat-inactivated Enterococcus faecalis has beneficial effects on gastrointestinal functions thus exhibiting direct action of heat-inactivated microbes on the gut function (Sawada et al., 2015).

Recently, more studies on human subjects have investigated the role of paraprobiotics. For instance, Sugawara et al., (2016) investigated the role of a heattreated strain of Lactobacillus gasseri given daily in a beverage to get $10^{10}$ cells per day, to a group of adults in a randomized placebo controlled study design, and noted the benefits as preventing the degeneration of quality of life and the occurrence of lifestyle-related diseases. In another randomized control trial on obese human subjects where a strain of heat-killed Lactobacillus amylovorus was given on a daily basis in a beverage for 12 weeks, a reduction of obesity was noted (Nakamura et al., 2016). With such findings, the impact of heat-killed microbes in cooked fermented products such as 'hoppers', 'thosai' and 'idli' are yet to be deciphered. Also in view of heat-killed microbes having shown positive results it may be necessary to recalculate the cell numbers of probiotics that should be present in the original food, based on specific beneficial effects they confer to the host.

\section{An overview of methods employed in probiotic food designing}

Considering the fact that fermented foods serve as probiotic carriers, we may categorize probiotic foods into three groups based on the state of the microbes and food matrix at the time of consumption: (1) the food matrix is fermented by the microbes in it as done in traditional methods and consumed without cooking (2) the food only serves as a vehicle to provide specific viable probiotic cells to the consumer, and (3) the fermented food is cooked before consumption, and therefore the microbes that helped in the fermentation are inactivated.

The $1^{\text {st }}$ and $3^{\text {rd }}$ groups represent traditional fermented foods which have the potential to deliver specific probiotics or specific paraprobiotics of interest respectively. The $2^{\text {nd }}$ group is well defined in delivering known probiotics of interest. In the traditional methods, depending on the type of food, often the introduction of microbial cultures is done by promoting the growth of the indigenous flora of the food ingredients. In making 'hoppers', coconut water is added to the rice flour dough, in making 'thosai', the indigenous flora of the black gram increase in number when the dough is made either mixing with water or coconut water and left overnight. In making 'kimchi' and 'sauerkraut' the food matrix is given a suitable salty anaerobic environment for promoting the growth of indigenous LAB making it acidic. However, in some fermented preparations the inoculant has to be made separately. For instance in making 'tempeh' (a soya bean product), a specific fungus (Rhizopus oryzae) has to be introduced as an inoculant, which involves a well spelt-out procedure.

When comparing the different groups, in the $1^{\text {st }}$ and $3^{\text {rd }}$, the action of the viable microbes eliminate metabolites to the food with a simultaneous partial digestion of its components. While some such metabolites are known to help improve nutritional attributes and promote health, partial digestion accomplished by the microbes can improve bioavailability of certain nutrients (Agnoli et al., 2017). With the knowledge that microbes in fermented foods introduce new compounds to the food, that are finally delivered to the gut (Marco et al., 2017) a shortcoming of the $3^{\text {rd }}$ group may be that some of the newly formed beneficial compounds cannot withstand the cooking temperatures. In spite of that a positive aspect of cooking is that the potential pathogens among the microbes will be killed before consumption. The advantages of using paraprobiotics are recorded as they can be easily processed and incorporated into various foods and beverages, and they exhibit a longer shelf life (Sugawara et al., 2016). The formulation of the $2^{\text {nd }}$ group is accomplished by using modern technology. The advantages of the $2^{\text {nd }}$ group are that specific probiotic strains with known beneficial effects can be incorporated to the food substrate without promoting fermentation. This novel method sans fermentation, necessitates the use of modern techniques like microencapsulation of probiotics (Shori, 2017), currently a technique that should be handled at the industrial level in mass scale production. One important advantage of the $2^{\text {nd }}$ group from the consumer's point of view is that the food in question is not altered. For this fact alone, the $2^{\text {nd }}$ group may be at an advantage because the technique of microencapsulation gives leverage to work with any type of food that is already popular. However healthy a new product may be, if it does not have the organoleptic appeal it is unlikely to be tried out by the consumer. Additionally, the method employed for the $2^{\text {nd }}$ group is well defined in terms of the probiotics and their numbers introduced. However, a major disadvantage of the $2^{\text {nd }}$ group is the absence of microbial action on the food matrix, and therefore the absence of beneficial compounds 
produced by the microbes.

\section{THE GUT MICROBIOTA MODULATED BY PROBIOTICS AND PREBIOTICS}

The microbial colonies present on and in the human body are commonly referred to as the microbiota. Over the initial years of life, the human body including the skin surface, oral cavity, and gut are colonized by an enormous variety of bacteria, archaea, fungi and viruses (mainly macrophages), which form a community - the human microbiota (Morgan et al., 2013; Viggiano et al., 2015). The gut microbiota with approximately $3 \times 10^{13}$ bacterial cells represents a significant proportion of the human microbiota (Roy and Trichieri, 2017).

The gut microbiota has been shown to have a significant influence on the health and wellbeing of the host; the latter part of this review is devoted to their health promoting aspect. However for maintenance of health, homeostasis of the microbiota plays an important role and disruption of this balance results in dysbiosis leading to ill health. Marinelli et al. (2017), report that invading pathogens, antibiotics, certain dietary factors, and smoking, may alter the balance of the microbiota. Such disruptions of homeostasis can lead to the emergence of opportunistic enteric pathogens such as Clostridium difficile (Aziz et al., 2013) an organism much discussed currently with regard to antibiotic resistance.

The intricacies of the influence of human microbiome (genes of the microbiota) on the human metabolism were unraveled in the recent past leading to the establishment of the Human Microbiome Project reference database (https://commonfund.nih.gov/hmp/index; The Human Microbiome Consortium, 2012), as well as other countrywise databases of similar nature. Scientists have deemed these microbes appropriately as the 'forgotten organ' (Willyard, 2011) for the enormous influence they have on the wellbeing of the host. This 'organ' composed of large numbers of microorganisms have their own survival mechanisms within the gut. They are divided into two groups based on their habitat, where some are considered resident (autochthonous) while others are introduced (allochthonous). The food we consume is presumed as the main route of entry for allochthonous microbes which may eventually play roles as probiotics, commensals, and pathogens.

\section{A survey of probiotic taxonomy}

Traditionally fermented foods may have an abundant number of microorganisms of which some having probiotic potential. The probiotics obtained from food are expected to modulate the microbiota. In that aspect, being knowledgeable on the composition of the gut microbiota is essential in designing probiotic foods. However there is a drawback with regard to the knowledge on the gut microbiota as the focus has been on entire ecosystems of bacteria and therefore individual species are not identified (Bourzac, 2014). This is because many bacterial species in the intestinal tract cannot be cultured in the laboratory. What is known is that on the whole the human gut microbiota is dominated by five bacterial phyla (Bacteroidetes, Firmicutes, Actinobacteria, Proteobacteria and Verrucomicrobia) and one Archaea (Euryarchaeota) (Tremaroli and Bäckhed, 2012). Also, it is known that about $90 \%$ of the bacterial phylotypes of the human microbiota belong to Bacteroidetes and Firmicutes (Cani and Delzenne, 2011; Louis et al., 2014). Table 1 lists some of the common genera representative of the gut bacteria within these phyla.

Even within a species of a genus, only some strains have probiotic potential. However in this review, names of strains within species are not mentioned, giving precedence to touch upon more general issues with regard to probiotics. With that understanding, the two most well known and widely used genera in probiotic foods are Lactobacillus and Bifidobacterium (Tremaroli and Bäckhed, 2012). Many of the probiotic species that respond to changes in carbohydrate intake seem to belong to the Firmicutes and Actinobacteria, which are considered as nutritionally specialized (Louis et al., 2014). Daniluk et al. (2012), have listed the most common probiotic bacterial species used in experiments, and among them are Lactobacillus spp. ( $L$. casei, L. paracasei, L. acidophilus, L. rhamnosus, L. brevis, L. plantarum, L. delbrueckii, L. gaserri, L. helveticus)

Table 1: Major phyla, and genera represented in the gut microbiota.

\begin{tabular}{|c|c|c|}
\hline Phyla & Gram reaction & Genera \\
\hline Bacteroidetes & Negative & $\begin{array}{l}\text { Bacteroides } \\
\text { Flavobacterium }\end{array}$ \\
\hline Firmicutes & Positive & $\begin{array}{l}\text { Lactobacillus } \\
\text { Staphylococcus } \\
\text { Streptococcus } \\
\text { Clostridium } \\
\text { Enterococcus }\end{array}$ \\
\hline Actinobacteria & Positive & $\begin{array}{l}\text { Bifidobacterium } \\
\text { Propionibacterium } \\
\text { Corynebacterium }\end{array}$ \\
\hline Proteobacteria & Negative & Escherichia \\
\hline Verrucomicrobia & Negative & Akkermansia \\
\hline
\end{tabular}


and Bifidobacterium spp. (B. bifidum, B. infantis, $B$. adolescentis, $B$. longum, $B$. breve. Lately many yeasts also have earned probiotic status (Mcfarland, 2015) with them being recorded in relative abundance in the gut (Iannitti and Palmieri, 2010; Lozupone et al., 2012). Specifically Saccharomyces boulardii is a yeast having probiotic effects (Cascio et al., 2013).

The Bacillus spp. too belong to Firmicutes just like the popular probiotic genus Lactobacillus. This genus stands out among the Gram-positive bacteria by its ability to produce heat stable endospores. Bacillus spp. are considered allochthonous and enter the gut by association with food (Hong et al. 2005). However, many Bacillus spp. are known to be enteropathogenic. A probiotic Bacillus subtilis has been recorded by Permpoonpattana et al. (2012), which has remained viable in baked foods because of their heat stable endospores. In fact, probiotic products containing Bacillus species are recorded to have been in the market for at least 50 years (Naidu et al., 2012).

Also Streptococcus is a genus with many pathogenic strains, but $S$. salivarius subsp. thermophilus is recorded to have probiotic effects (Daniluk et al., 2012). Among the Enterobacteriaceae (a family with many pathogenic genera), Eschericha coli-E. coli Nissle 1917 is recorded to have probiotic effects. Particularly, Escherichia coli (E. coli strain Nissle 1917) has been the subject of several investigations on probiotic mechanisms (Altenhoefer et al., 2004; Trebichavsky et al., 2010) and is used in a commercial product called Mutaflor capsules (marketed by Ardeypharm) (McFarland, 2015). This strain was originally isolated by Alfred Nissle (1874-1965) in 1917 while searching for $E$. coli wild-type strains with antagonistic activity against enteric pathogens, and it is shown to produce microcins (Altenhoefer et al., 2004), which are bacteriocins (antimicrobial metabolites). Additionally, the more recently isolated autochthonous Gram-negative organism, Akkermansia muciniphila may turn out to be useful in food applications. The health beneficial role of this species is currently getting established as it is recorded to have anti-inflammatory effects (Zhou, 2017). Lozupone et al. (2012) have recorded some names of probable core set of bacteria in the adult gut as Faecalibacterium prausnitzii, Roseburia intestinalis, and Bacteroides uniformis and among the archae are Methanobrevibacter smithii. However, Lozupone et al. (2012), also express the idea that the notion of the existence of a core set of organisms has been weakened with more information available.

\section{Health promoting metabolites and mechanisms within the gut}

The microbial metabolites formed within the gut have the potential to serve as energy sources, micronutrients, and health promoting substances. However, the enormous diversity of these metabolites remains largely unexplored (Degnan et al., 2014). As discussed, phytic acid is associated with dietary fiber and is considered as an antinutritional substance particularly in cereal based diets (even though it is noteworthy that phytic acid is considered as a functional food in certain diets worldwide) as it chelates with metal micronutrients particularly iron and zinc, compromising their bioavailability. A widely known method to breakdown phytic acid is by the use of microbial phytase (another method being activating endogenous phytase through soaking and sprouting beans before consumption) (Gibson and Ferguson, 1998). Therefore, introducing probiotics with phytase activity will serve to be beneficial in the rice based Sri Lankan diet.

To select a few other compounds of interest, two specific metabolites that deserve further discussion with regard to the current topic are short chain fatty acids (SCFA) and conjugated linoleic acid (CLA). The positive effects of host beneficial SCFA such as acetate, propionate, and butyrate production are many. The SCFAs may not only exert immunomodulatory effects but also trigger a decrease in the $\mathrm{pH}$, thereby altering the colonic environment (FrancoRobles and López, 2015). Butyrate is produced by members of Bacteroidetes (Thomas et al., 2011) and has been suggested to reduce inflammatory responses in the colon (Oh and Segre, 2013). Foods mainly composed of cereals, legumes, and vegetables seem to favour Bacteroidetes as they can thrive on non-digestible polysaccharides in them (Thomas et al., 2011). According to Gong et al., (2018) certain studies have traced the biochemical pathways influenced by the SCFAs exerting protective effects against host obesity and metabolic syndrome.

The SCFAs additionally have antioxidant potential. This can have a significant impact on curbing many NCDs for which a triggering factor is the occurrence of free radicals within the body. It is recorded that we consume about $3.5 \mathrm{~kg}$ of oxygen each day and when not completely reduced, the formation of free radicals and other reactive oxygen species (ROS) such as the radicals superoxide $\left(\mathrm{O}_{2}^{-}\right)$ and hydroxyl $\left(\mathrm{OH}^{-}\right)$occurs rapidly (Shahidi, 2004). Many NCDs are implicated to ROS as they can cause damage to proteins, lipids, carbohydrates and to DNA (Gill and Tuteja, 2010). While some ROS are released by the GI tract itself, some are present in the food, and yet others are generated by chemical reactions of dietary components within the stomach (Halliwell, 2007). Specially, such high oxidative stress level is related to a predisposition for diabetic complications (Qin et al., 2012). However, a positive attribute of the Sri Lankan diet and that of other Asian countries is the high intake of spices on a daily basis, which can augment the antioxidant capacity of the diet.

Interestingly, the occurrence of high levels of fructans in plants as well as in the gut (serving as prebiotics) is correlated with antioxidant capacity (Franco-Robles and López, 2015). Antioxidant rich food sources such as fruits and vegetables, undoubtedly confer a protective effect on the gastrointestinal tract which is constantly exposed to reactive species (Halliwell, 2007). Therefore, a diet rich in fruits and vegetables plays an important role in maintaining good health on their own accord. Having recognized their benefits a minimum of $400 \mathrm{~g}$ of fruit and vegetables per day (excluding potatoes and other starchy tubers) is recommended for the prevention of NCDs in addition to other benefits conferred by them (WHO, 2013). Relating this information to the present topic, the role the indigestible carbohydrates in the fruits and vegetables provide in 
modulating the gut microflora serving as probiotics, will in no doubt enhance the health benefits they confer on the host.

Also as mentioned, attributable to certain gut bacteria is their ability to produce health promoting CLA, which has attracted attention of nutritional scientists because of anti-inflammatory effects shown in several animal studies. CLAs are isomers of octadecadienoic acids. They are excellent antioxidants because of the conjugated bonding pattern in their chemical structure. Foods of ruminant origin generally contain more CLA than foods of non-ruminant origin due to the presence of linoleic acid isomerase in ruminal bacteria that catalyzes the isomerization of linoleic acid into CLA (Akalin et al., 2007). CLA is synthesized in the rumen as an intermediate by Butyrivibrio fibrisolvens while some Lactobacillus species also produce significant amounts (Nakamura and Omaye, 2012). There have been efforts to look for conditions suitable for CLA production in fermented foods. Dairy products, mainly yoghurts have been under scrutiny for CLA production due to the action of probiotics. In a recent study by Vieira et al., (2017) the need to pre-select LAB that are capable of producing CLA is noted because dietary CLA intake is generally low. Further, incorporation of fructooligosaccharides to yoghurt has shown to increase CLA production by the action of microbial fermentation (Akalin et al., 2007).

The members of the gut microbiota assist in the absorption of vitamins and minerals (Phillips, 2013). The role of the microbiota in producing essential vitamins became clearer when germ free animal experiments were performed, and as a result, specifically vitamins $\mathrm{K}, \mathrm{B}_{1}, \mathrm{~B}_{6}$, $\mathrm{B}_{7}, \mathrm{~B}_{9}$ (folic acid) and $\mathrm{B}_{12}$ production in the gut is reported (Degnan et al., 2014). Also, the ability of fermented foods to provide these vitamins due to microbial action has been widely documented. However, relying on the gut microbiota for supplementation of vitamin $B_{12}$ seems to be questionable according to Degnan et al. (2014) who argue that although it is synthesized by some human gut microbes, it is a precious resource within the gut itself and therefore is likely not provisioned to the host in significant quantities. Vitamin $B_{12}$ being one of the heat stable B group vitamins, it is likely that it will be available even in cooked fermented foods provided that the right microorganisms are there. For instance, at least one strain of the probiotic species Lactobacillus reuteri is known to produce vitamin $\mathrm{B}_{12}$ (Eck and Friel, 2013). Harnessing this effect in cooked fermented foods will be useful for a primarily vegetarian diet.

The digestive enzymes of the host could be affected by probiotics, a phenomenon that can be manipulated to reduce intestinal disease symptoms. In fact modulation of bacterial enzyme activity has been described as one of the well known mechanisms through which introduced preand probiotics exert their beneficial effects on the host (De Preter et al., 2008; Franco-Robles and López, 2015). This phenomenon has helped some individuals to deal with lactose intolerance from perhaps when health beneficial roles of fermented milk were realized, through the empirical knowledge gained. There are many other beneficial effects conferred by bacterial enzymes produced by the gut microflora, and many digestive and extradigestive diseases are linked to alteration of gut microbiota (Viggiano et al., 2015).

Antitoxic effects of the gut microbes are beneficial for the host as well as for the survival of the gut flora. For instance, alleviation of toxic effects including heavy metal toxicity is attributed to probiotics (Foligne et al., 2013; Cerbo et al., 2015). Furthermore the protective roles of probiotics on various mycotoxins (fungal toxins) have been highlighted (Taranu et al., 2010; Willyard, 2011; Cerbo et al., 2015) which by no means is attractive for the local scenario where lack of optimal storage conditions to various edible seeds, make them susceptible to fungal growth. Mycotoxins can get onto human food by the infestation of fresh produce by fungal pathogens. Binding of certain mycotoxins to probiotics correlates with reduced mutagenicity of these toxins (Oelschlaeger, 2010). In an in vitro study, two strains of lactobacilli have exhibited antiproliferative effects by diminishing apoptosis (programmed cell death) induced by a mycotoxin (ochratoxin A) (Cerbo et al., 2015).

\section{Promoting homeostasis within the microbiota}

As mentioned earlier, homeostasis within the microbiota is essential for maintaining health status of the host. A multitude of factors have been named as deciding factors for the composition of the microbiota with the daily diet being one of the most influential. McFarland (2015) lists five properties expected in a probiotic viz., survival to the target organ (in the present context to the colon), interaction with host systems, antipathogenic actions, safety and manufacturing concerns. The microbial mechanisms and key metabolites that shape the composition of the human gut microbiota are largely unknown, impeding efforts to manipulate dysbiotic microbial communities toward stability and health (Degnan et al., 2014). Making matters more complicated, it has been shown that the composition of the microbiota differs even within healthy individuals (The Human Microbiome Project Consortium, 2012). Taking these factors into consideration, probiotics taken in as food may serve to be autochthonous or allochthonous within the gut and it is probably not possible to strictly divide probiotics based on their habitat, and the concept of being autochthonous or allochthonous may be dependent on the individual rather than on the population at large. Appropriately, Hutkins et al. (2016) report that many different microbiota species, each with a potentially different utilization strategy, is likely to compete for prebiotic substrates in each individual, making it challenging for researchers to determine specifically which pathways affect the gut microbiota and why certain groups respond.

In spite of such ambiguity in the understanding on factors governing homeostasis within the gut, empirical awareness on health beneficial roles of traditional fermented foods backed with information from recent case control studies on probiotic effects have given the cue to proceed. The recently accumulated knowledge on paraprobiotics adds another dimension to the understanding 
of the mechanisms involved, and very soon with the accumulation of knowledge on paraprobiotics, it may be necessary to revise the current guidelines on probiotics and the way we perceive them.

\section{Harmful metabolites of the gut flora and beneficial effects of probiotics}

The capability of probiotics to govern homeostasis within the members of the microbiota keeping pathogens under control, is well established. However, at a practical level uncooked and excessively handled foods also will serve as vehicles to introduce a vast range of microbes to the microbiota. Therefore, as discussed earlier it is not only probiotics that will enter through the oral cavity along with food and not all bacteria in food will have a positive effect on the health of the consumer. Focusing on some negative aspects of the gut microbiota, not all their metabolites are health promoting. Microbial metabolites like branched chain fatty acids (Rehman et al., 2012) and secondary bile acids, promote carcinogenesis (Louis et al., 2014). Ammonia formed due to protein fermentation at harmful levels may result in the production of toxic metabolites; a much discussed topic in probiotic research on farm animals with regard to bacterial urease activity (Iannitti and Palmieri, 2010).

Not only bacterial metabolites but also some bacterial cell components affect the health of the host. Among them are the levels of lipopolysaccharides (LPS) that induce inflammation and endotoxiaemia which are closely linked to obesity and type II diabetes (Nakamura and Omaye, 2012). While Gram-negative bacteria have LPS as components of their cell membrane, Gram-positive bacteria have lipoteichoic acid in their cell membrane. Both these are known to elicit immune responses (Foligne et al., 2013).

The gut flora can produce mutagens by various means such as reacting with bile acids (Cerbo et al., 2015). Additionally certain microbial enzymes, such as some glycosidases (ß-glucuronidase and $ß$-glucosidase) produced by certain intestinal flora are known to have carcinogenic potential (De Preter et al., 2008) and these enzymes may interfere with the detoxification and excretion of toxic xenobiotics, such as drugs and environmental pollutants (Louis et al., 2014). An increase in Bifidobacteria in the gut flora in a randomized cross over study conducted by De Preter et al. (2008) has shown to be accompanied by a decrease in bacterial species that possess high $\beta$-glucuronidase activity. There is a keen interest to alleviate the effects of this microbial enzyme (Baharudin, et al., 2017; Roy and Trichieri, 2017). In fact, stimulation of Bifidobacteria has been shown to prevent the growth of most pathogens and putrefactive bacteria (Iannitti and Palmieri, 2010).

\section{Controlling pathogenic effects by probiotics}

The pathogens cause disease either by infections or by releasing toxins or by a combination of both. However, pathogens are a part of the microbiota surviving with commensals and probiotics in healthy individuals. This is confirmed in a map of the human microbiome (Morgan et al., 2013). Potential pathogens co-exist along with the rest of the microbiome as long as homeostasis is maintained. For instance, Staphylococcus aureus is a species known to cause food borne bacterial intoxications and in spite of that it is a part of the flora of the skin and the nasal cavity of humans. Nowrouzian et al. (2015), report that $S$. aureus has been identified as a common member of the gut flora of Swedish infants.

This trend of tolerating pathogens may be universal in living systems. For instance, such a homeostatic balance has been shown in plants too, where studies on plants have shown that high fitness of the plant host leads to a reduction of pathogen burden and tend to tolerate existing pathogens strategically (Chervonsky, 2012). In fact, Hacquard et al. (2015) have reported on common features of the gut and plant microbiota in preventing pathogen establishment, which sheds light into their protective roles of competitive exclusion against invading pathogens. Such comparative information between plants and humans is important as ultimately it is the same naturally present microbes that find their way to the human alimentary canal when plants serve as food. Being aware of the mechanisms will help in avoiding their harmful effects leading to disease. However, given the complexity of the microbiota and its effects on the immune responses, the mechanism by which the normal microbiota directly limits the growth of pathogens is not fully understood and it may differ between infections (Jarchum and Pamer, 2011).

Considering the prevalence of pathogens on fresh foods and unhygienic handling of foods being rampant according to the media, the number of reports of food poisoning is not that great. In fact, Whipps et al. (2008) express surprise why more outbreaks of food poisoning do not occur worldwide, attributing this tolerance to the cell numbers that should be ingested to cause disease (infectious dose). It is suggested that intestinal pathogens such as Salmonella typhimurium submit to colonization resistance provided by the microbiota preventing systemic infection (Chervonsky, 2012). In this regard probiotics within the gut flora seem to serve an exceptional role in controlling pathogens.

It is however recorded that infections can be caused by as few as 800 Campylobacter cells, 100 cells of $E$. coli O157:H7 and Shigella spp., and fewer than 100 particles of norovirus (Whipps et al., 2008). It will be interesting to determine whether these figures are linked to the composition of the gut flora and/or that of the diet. Probiotic effectiveness has been thought to be dependent on their ability to compete with other organisms. However, with paraprobiotics exhibiting unanticipated roles, the complex nature of colonization resistance exerted by the microbiota on incoming pathogens is yet to be ascertained.

It is obvious that the gut microflora play a complex role in warding off ingested pathogens. Within the gut of a healthy host, colonization resistance is exerted by the microbiota on incoming pathogens by a number of mechanisms; by competing for limiting resources and physically occupying pathogen attachment sites by exerting anti-adhesive, anti-invasive and antitoxin effects, 
by producing antimicrobial substances, by modulating the immune system and by strengthening the gut epithelial barrier (Oelschlaeger, 2010). The gut barrier balances the interactions of microorganisms and nutrient compounds and is composed of two components (physical barrier surface, which prevents bacterial adhesion and regulates paracellular diffusion to the host tissues and a functional barrier which discriminates pathogens) (Viggiano et al., 2015).

Additionally, the immune system plays a major role in striking a balance between being tolerant to the microbial communities within and efficiently responding to infection by exogenous pathogens (Jarchum and Pamer, 2011). When a pathogen appears and multiplies the microbiota provides both pro and anti-inflammatory signaling required to support homeostasis (Chervonsky, 2012). When certain microbes are too numerous leading to dysbiosis, the immune system becomes activated and triggers inflammation (Bourzac, 2014) and when the microecology is altered the gut barrier may be affected (Viggiano et al., 2015) thereby leading to ill-health. Whether dysbiosis is linked to disease onset or whether disease causes dysbiosis is yet to be found out. However what seems to be important with regard to the current topic is that timely introduction of probiotics has helped the microbiota back to homeostasis (Bourzac, 2014). In this regard, Jarchum and Pamer (2011) have mentioned the importance in identification of microbial species that affect the immune response and the mechanisms they employ to maintain immune homeostasis. Nevertheless, with the unraveling of the beneficial roles played by paraprobiotics, the mechanisms involved have become elusive.

\section{Health beneficial effects conferred on the host by probiotic bacteria}

Overall, the effects of probiotics may be prophylactic or therapeutic and they influence positively on a wide range of diseases from infections to NCDs with evidence also building up that they could even help in the therapy of psychological disorders (Table 2). Based on the effort of the International Life Sciences Institute (www.ilsi.org), Coman et al. (2012) reported evidence of functionality of probiotics on human applications over a broad scale, viz., metabolism, chronic inflammatory and functional disorders, infections and allergies. If nasal blockage caused by phlegm in preschool children due to the consumption of milk (mentioned before) is an allergic condition, incorporation of specific probiotics may help alleviate this problem.

\section{Influence on host gene expression -combating diseases}

Specific commensal bacteria are recorded to impart different signaling patterns on the immune response to invading pathogens based on their class and anatomical location of

Table 2: An overview of positive roles played by probiotics on different maladies.

\begin{tabular}{|c|c|}
\hline Malady & Reference (direct or quoted therein) \\
\hline Type II diabetes control & Qin et al. (2012); Ruan et al. (2015); Cerbo et al., (2015) \\
\hline Cholesterol lowering effects & $\begin{array}{l}\text { Change et al. (2011); Jones et al. (2012); Foligne et al.(2013); Fuentes et al. } \\
\text { (2013); Cerbo et al. (2015); Ruan et al. (2015); Marco et al. (2017) }\end{array}$ \\
\hline Anticancer effects & Daniluk et al., (2012); Marinelli et al. (2017); Roy and Trinchieri (2017) \\
\hline $\begin{array}{l}\text { Prevention of diarrhea of viral and } \\
\text { bacterial origin, and antibiotic- } \\
\text { associated }\end{array}$ & $\begin{array}{l}\text { Lozupone et al. (2012); Rehman et al. (2012); Daniluk et al. (2012); Ray et } \\
\text { al. (2016); Marco et al. (2017) }\end{array}$ \\
\hline $\begin{array}{l}\text { Ease symptoms of inflammatory } \\
\text { bowel disease }\end{array}$ & $\begin{array}{l}\text { Lozupone et al. (2012); Abt and Artis (2013); Bron et al. (2013); Taverniti } \\
\text { and Guglielmetti (2011); Iannitti and Palmieri (2010) }\end{array}$ \\
\hline $\begin{array}{l}\text { Ease gastritis associated with } \\
\text { Helicobacter pylori }\end{array}$ & Cerbo et al. (2015); Iannitti and Palmieri (2010) \\
\hline $\begin{array}{l}\text { Inhibition of microbial pathogens and } \\
\text { influence on host immune response }\end{array}$ & $\begin{array}{l}\text { Altenhoefer et al. (2004); Abt and Artis (2013); Saad et al. (2013); McFarland } \\
\text { (2015); Chervonsky, (2012) }\end{array}$ \\
\hline Alleviation of lactose intolerance & Ray et al. (2016); Granato et al. (2010); Tripathi and Giri (2014) \\
\hline Alleviation of certain allergies & Taverniti and Guglielmetti (2011); Koplin et al. (2018) \\
\hline Obesity control & $\begin{array}{l}\text { Tsukumo et al. (2009); Oh and Segre (2012); Abt and Artis (2013); Karlsson } \\
\text { et al. (2013); Foligne et al. (2013); Bourzac (2014); Marco et al. (2017); } \\
\text { Torres-Fuentes et al. (2017) }\end{array}$ \\
\hline $\begin{array}{l}\text { Influence on mood and anxiety } \\
\text { disorders }\end{array}$ & $\begin{array}{l}\text { Foster and Neufeld (2013); Marco et al. (2017); Torres-Fuentes et al. (2017); } \\
\text { Bailey and Cryan (2017) }\end{array}$ \\
\hline $\begin{array}{l}\text { Ease disorders involving } \\
\text { neurodevelopment, including autism }\end{array}$ & Finegold (2012); Gilbert et al. (2013) \\
\hline
\end{tabular}


the infection (Abt and Artis, 2013). This has a protective effect against infections. Amazingly even the severity of an influenza infection has been found to be dependent on the gut microbiota and the mechanism of protection appears to be linked to the activation of inflammasome (component of the innate immune system) as reported by Chervonsky, (2012) citing other studies. Also, research has indicated that susceptibility to some of the NCDs is linked to the role of the metagenome (The Human Microbiome Project Consortium, 2012). For instance, the risk of developing type II diabetes may also involve factors from the gut metagenome (Qin et al., 2012). These phenomena give an indication of the influence the gut flora have on the host genes.

In the process of providing important biological and metabolic functions that cannot be performed by the human metabolism alone, interactions between host-microbe genetics and the environment appear to be intricate, complex (Cani and Delzenne, 2011), and simply overpowering. Hence this entity of genes from the microbiota is collectively called the 'metagenome' (Oh and Segre, 2013). The Human Microbiome Project, using metagenomic analysis has identified $>40,000$ species in the colon and is in the process of creating profiles of the normal microbiological constituents found in healthy humans (McFarland, 2015). Amazingly the microbiome has been noted to encode a consortium of genes exceeding the human genome by a magnitude of about 150 (Cani and Delzenne, 2011; Velasquez-Manoff, 2015) showing the immense influence that microbial genes have on the host. As discussed previously Lozupone et al. (2013) express skepticism on the existence of a core set of organisms. In spite of that even recent publications such as that of TorresFuentes et al. (2017), still refer to a core set of genes. It is reported that the gut microbiota can vary over a 10 -fold range among individual humans in their relative abundance of species but at the level of gene functions, less variability is observed among individuals pointing to the existence of a conserved functional core (Hacquard et al., 2015).

While the roles played by consumed probiotics, which acclimatize into the microbiota affecting host's health, spans a wide assortment, how paraprobiotics exert similar effects is elusive. In spite of the fact that they are dead, the cell components of paraprobiotics ingested by the host seem to influence the immune system (Taverniti and Guglielmetti, 2011). One key role of heat killed cell components appear to be in their ability to block important signal transduction pathways associated with pathogenesis (Sang et al., 2015). However, the exact mode of the activity of paraprobiotics is yet to be ascertained (Sawada et al., 2016; Nakamura et al., 2016).

\section{Role of probiotics in combating specific NCDs}

Reduction of serum cholesterol and anticarcinogenic activity are specific prophylactic effects attributed to probiotic action. Certain lactobacilli are found to be capable of competitively inhibiting carcinogen and mutagen formation (Cerbo et al., 2015). Some probiotics modulate cell proliferation and apoptosis which are mechanisms pointing towards potential cancer therapy, but their effects seem to vary depending on factors like the cell type on which they act (Daniluk et al., 2012). Hitherto, anticancer therapeutic effects of several probiotics have been tested on animal models which have given encouraging results, however, the effects on humans are yet to be ascertained (Marinelli et al., 2017). Also, in spite of a diet and lifestyle prone to obesity some individuals are less susceptible to weight gain. Based on this observation Tsukumo et al. (2009) express that mechanisms which are not directly related to the human genome may be involved in gaining weight. Probiotics are known to upregulate expression of proglucagons and decrease adiposity (Asemi et al., 2013). [Glucagon is a hormone that helps in regulating the appetite.]

Currently there is intense interest in the manipulation of the gut microbiota in controlling obesity (Table 2). Changes in the composition of the gut microbiota have been observed in people who are obese, and conversely the microbiota can respond to changes in body weight (Tremaroli and Bäckhed, 2012). Focusing on specific microbes in the gut, the population of Bacteroidetes and Firmicutes has been reported to be crucial in the development of obesity and diabetes. A relative abundance of Bacteroidetes has been shown to be higher in diabetic-prone rats (Nakamura and Omaye, 2012). A change in the proportions of Bacteroidetes to Firmicutes has been attributed to obesity in humans (Tsukumo et al., 2009). Additionally it has been noted that obese subjects hold lower bacterial diversity in the gastrointestinal tract compared to lean subjects and A. muciniphila-like bacteria have been more abundant in children with normal body weight compared to children with excessive body weight (Karlsson et al., 2012). Also Nakamura and Omaye (2012) indicate that while the genus Lactobacillus beneficially affects the host, different species of this genus dominate in the microbiota of obese individuals viz., high levels of Lactobacillus casei/paracasei and Lactobacillus plantarum in human gut are negatively associated with obesity and high levels of Lactobacillus reuteri are positively associated. Additionally, certain lactobacilli are also known to have inhibitory activity against intestinal enzymes involved in hydrolysis of carbohydrates thus promising a novel therapeutic tool for the treatment of diabetes (Cerbo et al., 2015).

Probably because Gram-negative bacteria have high levels of LPS in their cell membranes, it is recorded that the Gram-negative to Gram-positive ratio influences fat mass, body weight, insulin resistance and diabetes (Tsukumo et al., 2009). It is also known that SCFA are able to suppress the growth of Gram-negative bacteria, thus having a check on the LPS production. Asemi et al. (2013) have stated that using glucose as their primary energy source probiotics are influential in maintaining serum insulin levels. Adding to this finding is that modification of gut microbiota by probiotic supplementation may be a method for preventing and controlling hyperglycemia in clinical practice (Ruan et al., 2015).

Many Lactobacillus spp. are known to reduce tendency for cholesterol deposition. The biochemistry involved in 
the reduction of cholesterol levels by probiotics has been hypothesized. For instance, the ability of Lactobacillus reuteri to reduce serum cholesterol is known to be linked to high bile salt hydrolase activity affecting the host's bile salt metabolism, with the formation of deconjugated bile acids (Jones et al., 2012) which finally may co-precipitate with cholesterol and be excreted with the feces. Fuentes et al. (2013) collated such information citing other authors specifically on the activity of different strains of Lactobacillus plantarum. Among the ideas presented are that bacterial bile salt hydrolase activity may reduce plasma cholesterol through the reduction of enterohepatic circulation of bile salts, thus reducing bioavailability of cholesterol from the diet. It is also noted that the organic acids produced by bifidobacteria may inhibit cholesterol production (Prasanna et al., 2014). Propionic acid produced by the bacteria can cause a decrease in the systemic levels of blood lipids by inhibiting hepatic cholesterol synthesis and/or redistributing cholesterol (Fuentes et al., 2013). These authors also mention that the production of butyric acid by $L$. plantarum helps by providing energy for the colonocytes. In addition to its action of bile salt hydrolase mentioned above, the bacteria may also directly remove cholesterol by either incorporating it into the bacterial cell membrane, binding it to the cell surface or assimilating it during growth (Chang et al., 2011).

In a recent review Bailey and Cryan (2017) stated that the key role of the microbiota on the host immune system has been known for several decades, but the concept that the microbiota plays a key role in programming many other systems in the body is relatively new. With the advancement of technology, genome-wide association studies have identified genetic variations that are common to individuals in populations with a specific NCD. Animal studies, later extended to humans have demonstrated the impact of probiotics and prebiotics on diseases involving the brain and behavior, although the effect has been more pronounced in laboratory animals than in human populations (Bailey and Cryan, 2017). Amazingly, the therapeutic effects of the microbiota appear to go beyond mere physical disorders extending to ease disorders involving cognitive defects, such as mood and anxiety disorders as well as in easing autism and other neurodevelopmental disorders (Table 2). The microbiota, through the gut-brain connection, has been suggested to play a crucial role in such disorders (TorresFuentes et al., 2017; Bailey and Cryan, 2017). It is startling that statistically significant differences between control and autistic children of different bacterial phyla in the fecal flora especially with the four phylae Bacteroidetes, Firmicutes, Actinobacteria and Proteobacteria have been observed (Finegold 2011) indicating the close link between the composition of the microbiota and health status of the host.

\section{SPECIFIC ISSUES IN THE INTRODUCTION OF PROBIOTICS TO THE SRI LANKAN DIET}

Fermented foods and beverages are made through controlled microbial growth (Marco et al., 2017). Probiotic strains with known beneficial effects may be incorporated into such fermented foods subsequently. Therefore, an initial step is to popularize fermented foods. It is said that sometimes there is only a thin line between fermentation and food spoilage. A food that is considered spoilt by a certain population may turn out to be a fermented food source that is much relished by another population. Accustoming the consumer to this new group of food, specially when served uncooked, will initially be a difficult task. Taking the initial step to introduce cooked fermented foods will be a wise decision. Information should be available on issues relevant to specific groups of the population on the Sri Lankan scenario that will benefit by the introduction of fermented foods. Also, as fermented plant-based foods largely being a novel group of food, the need to look at safety issues is imperative. Related to this point, providing necessary guidelines for the introduction of such foods is also essential.

\section{Justification to incorporate fermented foods into the Sri Lankan diet}

With nutritional indicators remaining static compared to other health indicators (Ubeysekara et al., 2015), dietary diversification obviously seem to be an essential part of novel strategies to combat undernutrition. Specifically, the weaning period after exclusive breast-feeding is known to be a crucial period, as it is recorded that undernutrition initiates during this period and thereafter rises sharply in the second year of life (Ubeysekara et al., 2015). As the children grow up they depend heavily on unhealthy snack foods made of refined wheat flour (Karunaratne, 2007). In the adult diets, starch intake is reported to exceed the upper limit of the recommendations (Jayawardena et al., 2012).

Popularizing fermented foods is seen as a feasible suggestion to improve the diet of preschool children with the observation of the absence of a significant amount of fermented foods in their diet, except rarely consumed 'hoppers' (Karunaratne, 2007). Changing the method of processing has shown to improve nutritional quality. For instance, while soya textured vegetable protein (TVP) record high levels of phytic acid (Karunaratne et al., 2008), 'soya tempeh' and 'soya tofu', report progressively lower levels of phytic acid (Seneviratne et al., 2011). While 'soya tofu' is not fermented, the fermentation process of 'soya tempeh' is through the use of a fungus, which involves a well spelt out protocol. 'Soya tempeh' is known to be a good source of vitamin $B_{12}$ too (Watanabe et al., 2014).

The nutritional and health promoting roles of probiotics in the daily diet are well known in spite of limitations in understanding the mechanisms involved. In spite of pulses having many healthy attributes such as serving as choices for improving protein quality in a vegetarian diet (Agnoli et al., 2017) regrettably, they are reported to have high levels of phytic acid with the level dependent on processing methods employed (Seneviratne et al., 2011). Soya TVP which is a popular form of protein as consumed locally, is known to be high in iron, but unfortunately high in phytic acid too, thus impairing iron absorption (Karunaratne et al., 2008).

By incorporating fermented foods, the microbes in them are expected to reduce the level of phytic acid of the 
food matrix, through the production of microbial phytase. The ability of probiotics in curbing diarrheal diseases is recorded in several studies (Table 2), which can be perceived as another beneficial effect of adding probiotic rich foods to the daily diet in view of the fact that diarrheal diseases being identified as a common cause of undernutrition in the age group of 6-24 months (Ubeysekara et al., 2015).

\section{The need to move forward with caution}

In spite of the projected benefits, there are issues of concern. For instance, the tropical environment of the country is conducive for the survival of microbes in high numbers in fresh produce and stored food. Such environmental conditions call for stringent food handling and storage practices. The prevalent conditions in certain retail outlets and eateries are far from satisfactory. In spite of that, the majority of the population is compelled to depend on commercially prepared food on a daily basis because of their busy life style.

In this backdrop of circumstances, there is a chance that the introduction of viable probiotics at a commercial food outlet level will increase the load of microbes added to the daily diet. The consequence may be potentially dangerous as the probability of the population not being able to tolerate any higher numbers of microbes present in the food is high. Although probiotics are beneficial, some microorganisms associated with them may be opportunistic pathogens (Panghal et al., 2018). In fact in some instances, even probiotic therapy has shown undesirable side-effects with the introduction of viable lactobacilli, as discussed by Cerbo et al. (2015). However, related to this idea, representing a completely different setting, it is interesting to note the comment of Marco et al. (2017) referring to the highly processed and sanitized foods consumed in Western societies where they argue that the consumption of fermented foods may be a beneficial way to get microbial exposure for the normal development of the immune system and neural function. Contrarily, in the Sri Lankan scenario, the benefits expected by introducing fermented foods to the daily diet are different and the relative lack of hygienic conditions should be taken into account, which may cause a threat.

In view of the risks involved, the introduction of fermented foods should be done progressively at different levels, viz. household level, cottage industry level, medium and large scale industries. Introducing cooked fermented foods will be a safer option at the beginning, than introducing uncooked fermented foods. This move can be later extended to introduce potential paraprobiotics with health beneficial effects. Novel fermented doughs that are converted to pancakes or steamed cakes will serve as means to achieving this objective. This preparatory method is already familiar to the local population, which is the method of preparation of 'hoppers', 'thosai', and 'idly'. Such a move will help in the incorporation of more variety to the daily diet. Most types of dough made with pulse based flours ferment on their own accord when the suitable conditions are provided. As mentioned earlier, the traditional use of coconut water to ferment dough will be acceptable to the local population. Therefore, such familiar ingredients and the familiar methods of preparation may be popularized with concurrent research on paraprobiotics that could be incorporated to this type of foods. When using cooked fermented foods, heat sensitive micronutrients that may be lost also need to be investigated and addressed appropriately.

At the beginning, fermented foods suiting the local palate can be gradually introduced at the family level through the mass media. Such a wide range of fermented products may diversify the diet increasing both balance and variety. This may help improve nutritional status of children. Also, there are a variety of fermented traditional weaning foods in other countries (Sahlin, 1999; Blandino et al., 2003; Marshall and Mejia, 2011). Innovative ideas of weaning foods suitable for the local population may be developed initially, adopting information already available from such countries that use them on a regular basis with their traditional knowledge. Such preparation methods of weaning foods can be tested and eventually the technology developed can be transferred to mothers/guardians.

A long term approach to address malnutrition among children and adults would be for the local industries to develop appealing hygienically packed fermented foods of plant origin, to suit the local palate. Introducing specific probiotic strains of interest to the newly introduced fermented foods can follow subsequently. Also, in addition to introducing a variety of fermented plant based products, there is a possibility that the repercussions of low intake of milk due to phlegm development by preschool children (Karunaratne, 2007) may be corrected by diversifying fermented milk products by adopting a variety of traditional methods of milk fermentations used in other countries, such as 'kefir' and 'ryazhenka' (from Russia), different types of sour creams and cheese.

\section{The necessity of guidelines and quality control protocols}

Proper guidelines and techniques with regard to the organisms and conditions used for fermentation are essential to prevent disastrous outcomes. Currently bacteria, yeasts, and filamentous fungi are used in food fermentations. These are the very organisms that can cause diseases and food spoilage if the correct organisms are not employed. Specially fungi which produce various mycotoxins (which are mostly heat stable) can be inadvertently added to food in the process of fermentation either as contaminants, or as inoculants through negligence. In spite of the positive facts discussed with regard to 'soya tempeh', it is not available in the local market probably for a good reason, because the preparation of the starter culture is complicated and may be dangerous if not done under proper conditions. The author recalls this product being available as 'soya karawela' about three decades back.

Having laid the foundation, probiotics with known beneficial effects can be incorporated to such fermented foods. Ascertaining the characteristics of the organisms used as inoculants is an important step in developing a probiotic market which should be done with well laid out quality control protocols. Fermentation technology 
could be incorporated to build up a healthy snack food market in the country. To introduce probiotic foods, much patronage to the industry, in terms of research is needed. When specific inoculants are required along with appropriate technology, stringent protocols are essential. For this reason introducing mass scale production of novel uncooked fermented foods should be an undertaking taken up by the local industries collaborating with institutes such as universities, government departments and co-operations, with the concurrence of the health authorities. Knowledge on microbiology and facilities to work with microbes are essential accessory requirements to support the local probiotic industry.

\section{Future research needs for expanding probiotic potential at a global level}

In spite of the shortcomings on the knowledge on the microbiota and its complexities within the gut environment, certain factors on harnessing its benefits are clear. The idea has been mooted to create designer probiotics harnessing the benefits of the natural probiotics (Singh et al., 2017). However, research investigations seem to suggest that instead of viable probiotics, ingesting paraprobiotics along with prebiotic rich foods will still be health beneficial.

Expanding on harnessing the benefits of probiotics, the promising roles of prophylactic and therapeutic effects displayed by dietary probiotics seems to suggest that the future of probiotic foods may lie in their ability to confer more precise health beneficial effects on the consumer. Already their ability to reduce negative effects of drugs and to enhance their effectiveness is being exploited. For instance, oral treatment with lyophilized live probiotics has shown to prevent intestinal toxicity in cancer patients treated with radiotherapy and chemotherapy (Roy and Trinchieri, 2017). Also, Marinelli et al., (2017) report of studies where certain strains of gut bacteria synergize with largely-used anticancer drugs as immune checkpoint blockade agents. Harnessing of the microbiome to develop new narrowspectrum antibiotics is yet another idea for future research (Aziz et al., 2013) so that antibiotic use could be targeted to the organisms of interest. It is hypothesized that some day patients will be prescribed a combination of probiotics and prebiotics (Willyard, 2011) completely replacing pharmaceutical drugs. This trend is, progressing beyond functional foods.

\section{CONCLUDING REMARKS}

As discussed in the present review some of the microbes in fermented foods serve as probiotics, and therefore increasing the choice of fermented foods in the Sri Lankan diet increases the chance of getting exposed to probiotics. With the Sri Lankan diet being primarily plant based, it has the potential of being rich in prebiotics because of the high levels of dietary fibre. However, considering that excessive levels of dietary fibre is associated with phytic acid, and certain food components that can reduce levels of phytic acid are not a part of the typical Sri Lankan diet, introducing fermented foods is envisaged as a simple way to improve the nutritional quality of the local diet. The unsatisfactory nutritional indicators of preschool children which remain static over several years, and the poor dietary habits of the Sri Lankan population at large, show that novel strategies should be tried out to improve the nutritional quality of the diet through dietary diversification. The gradual addition of fermented foods seems to be a good option to move forward while encouraging the consumption of sprouted seeds is also envisaged as helpful due to the presence of indigenous phytase enzyme that breaks down phytic acid.

Initially, considering the limitations specially with regard to inferior handling methods, adopting and introducing cooked fermented foods used in other countries using already familiar food ingredients appears to be the entry point to the fermented food technology. Such a move will gradually accustom the local palate to a wider range of cooked fermented foods using already familiar ingredients and techniques. Eventually, as the nutritional indicators provide more information on their effects, specific probiotic strains may be incorporated to such foods either as paraprobiotics or viable probiotics.

Food is obviously a straightforward factor that can be manipulated to promote homeostasis of the gut microbiota, which leads to good health. Therefore, such a move of introducing a wider variety of fermented foods, and eventually introducing probiotics through them, may be anticipated to alleviate some of the effects of antinutrients and add nutrients to the daily diet, while helping in fighting NCDs, through the boosting of the immune system. Whether the diet is well-balanced or not, the advantages of introducing fermented foods to the Sri Lankan diet seem to be rewarding. If specific benefits with regard to combating undernutrition are forthcoming, developing a local probiotic food industry will be a viable option. This way, known probiotics with specific benefits can be added to the daily diet. To realize this, scientists, industry, and the policy makers must work in unison.

\section{ACKNOWLEDGMENTS}

The author wishes to acknowledge the thought provoking discussions on the nutrition of the Sri Lankan diet with Dr. P.H. Amerasinghe, Dr. H.H. Sandstead, and Prof. P.A.J. Perera, my mentors in nutritional studies, which prompted ideas for writing this paper.

\section{REFERENCES}

Abt M.C. and Artis D. (2013). The dynamic influence of commensal bacteria on the immune response to pathogens. Current Opinion in Microbiology 16: 4-9.

Agnoli C., Baroni, L., Bertini, I., et al., (2017). Position paper on vegetarian diets from the working group of the Italian Society of Human Nutrition. Nutrition, Metabolism \& Cardiovascular Diseases 27: 10371052.

Akalin, A.S., Tokusoglu, O., Gonc, S. and Aycan, S. (2007). Occurrence of conjugated linoleic acid in probiotic yoghurts supplemented with fructooligosaccharide. International Dairy Journal 17: 1089-1095.

Altenhoefer, A., Oswald, S., Sonnenborn, U., Enders, C., Schulze, J., Hacker J., and Oelschlaeger TA (2004). The probiotic Escherichia coli strain Nissle 1917 interferes 
with invasion of human intestinal epithelial cells by different enteroinvasive bacterial pathogens. FEMS Immunology and Medical Microbiology 40: 223-229.

Asemi, Z., Samimi, M., Tabassi, Z., Rad, M.N., Foroushani, A.R., Khorammian, H., and Esmaillzadeh, A. (2013). Effect of daily consumption of probiotic yoghurt on insulin resistance in pregnant women: a randomized controlled trial. European Journal of Clinical Nutrition 67: 71-74.

Aziz, Q., Dore, J., Emmanuel, A., Guarner, F. and Quigley, E.M.M. (2013). Gut microbiota and gastrointestinal health: current concepts and future directions. Neurogastroenterology Motility 25: 4-15.

Baharudin, M. S., Taha, M., Imran, S., Ismail, N. H., Rahim, F., Javid, M. T., Muhummad, T. J.,Khalid, M.K. and Ali, M. (2017). Synthesis of indole analogs as potent $\beta$-glucuronidase inhibitors. Bioorganic Chemistry 72: 323-332.

Bailey, M.T. and Cryan, J.F. (2017).The microbiome as a key regulator of brain, behavior and immunity: Commentary on the 2017 named series. Brain Behavior Immunity 66: 18-22.

Blandino, A., Al-Aseeri, A.E., Pandiella, S.S., Cantero, D. and Webb C. (2003). Cereal-based fermented foods and beverages. Food Research International 36: 527-543.

Bourzac, K. (2014). Microbiome: The bacterial tightrope. Nature 516: S14-S16.

Cani, P.D., Delzenne, N.M. (2011). The gut microbiome as therapeutic target. Pharmacology \& Therapeutics 130: 202-212.

Cascio, V., Gittings, D., Merloni, K., Hurton, M., Laprade, D. and Austriaco, N. (2013). S-Adenosyl-L-Methionine protects the probiotic yeast, Saccharomyces boulardii, from acid-induced cell death. BMC Microbiology 13(35): 1-10. doi: 10.1186/1471-2180-13-35 Accessed June 1, 2016.

Cerbo, D.A., Palmieri, B., Aponte, M., Morales-Medina, J.C., Iannitti, T. (2015). Mechanisms and therapeutic effectiveness of lactobacilli. Journal of Clinical Pathology 0:1-17. Published online first doi:10.1136/ jclinpath-2015 202976 Accessed December 10, 2016.

Chang, B.J., Park, S.U., Jang, Y.S., Ko, S.H., Joo, N.M., Kim, S.I., Kim, C.H. and Chang, D.K. (2011). Effect of functional yoghurt NY-YP901 in improving the trait of metabolic syndrome. European Journal of Clinical Nutrition 65: 1250-1255.

Chen, X., Xu, J., Shuai, J., Chen, J., Zhang, Z., Fang, W. (2007). The S-layer proteins of Lactobacillus crispatus strain ZJ001 is responsible for competitive exclusion against Escherichia coli O157:H7 and Salmonella typhimurium. International Journal of Food Microbiology 115: 307-312.

Chervonsky, A.V. (2012). Intestinal commensals: influence on immune system and tolerance to pathogens. Current Opinion in Immunology 24: 255-260.

Coman, M.M., Cecchini, C., Verdenelli, M.C., Silvi, S., Orpianesi, C., and Cresci, A. (2012). Functional foods as carriers for $\mathrm{SYNBIO}{ }^{\circ}$, a probiotic bacteria combination. International Journal of Food Microbiology 157: 346-352.

Daniluk,U., Alifier, M. and Kaczmarski, M. (2012).
Probiotic-induced apoptosis and its potential relevance to mucosal inflammation of gastrointestinal tract. Advances in Medical Sciences 57(2), 175-182.

De Preter, V., Raemen, H., Cloetens, L., Houben, E., Rutgeerts, P. and Verbeke, K. (2008). Effect of dietary intervention with different pre- and probiotics on intestinal bacterial enzyme activities. European Journal of Clinical Nutrition 62: 225-231.

Demarigny, Y. (2012). Fermented food products made with vegetable materials from tropical and warm countries: microbial and technological considerations. International Journal of Food Science \& Technology. 47:2469-2476.

Donkor, E.S. (2013). Sequencing of Bacterial Genomes: Principles and Insights into Pathogenesis and Development of Antibiotics: Review. Genes 4: 556572.

Eck, P. and Friel, J. (2013). Should Probiotics be considered as Vitamin Supplements? Gastroenterology 138: 789791.

FAO/WHO (2001). Health and nutritional properties of probiotics in food including powder milk with live lactic acid bacteria, a joint FAO/WHO expert consultation. Cordoba, Argentina, 1-4 October, 2001. http://www. who.int/foodsafety/publications/fs_management/en/. Accessed July 23, 2013.

FBDG (2011). Food based dietary guidelines for Sri Lankans, $2^{\text {nd }}$ Ed. A publication by Nutrition Division of Ministry of Health of Sri Lanka in collaboration with the World Health Organization, http://www.fao.org/3/aas886e.pdf Accessed November 20, 2017

Finegold, S.M. (2011). State of the art; microbiology in health and disease. Intestinal bacterial flora in autism. Anaerobe 17: 367-368.

Foligne, B., Daniel, C., Pot, B. (2013). Probiotics from research to market: the possibilities, risks and challenges. Current Opinion in Microbiology 16: 284292.

Foster, J.A., Neufeld, K. M. (2013). Gut-brain axis: how the microbiome influences anxiety and depression. Trends in Neuroscience. 36: 305-312.

Franco-Robles, E., López, M.G. (2015). Implication of Fructans in Health: Immunomodulatory and Antioxidant Mechanisms. The Scientific World Journal. http:// dx.doi.org/10.1155/2015/289267 Accessed November $10,2016$.

Fuentes, M.C., Lajo, T., Carrion, J.M. and Cune, J. (2013). Cholesterol-lowering efficacy of Lactobacillus plantarum CECT 7527, 7528 and 7529 in hypercholesterolaemic adults. British Journal of Nutrition 109: 1866-1872.

Gibson, R.S. and Ferguson, E.L. (1998b). Assessment of dietary zinc in a population. American Journal of Clinical Nutrition 68 (Suppl:), 430S-434S.

Gibson, G.R. and Roberfroid, M.B. (1995). Dietary modulation of the human colonic microbiota: introducing the concept of prebiotics. The Journal of Nutrition 125: 1401-1412.

Gilbert, J.A., Krajmalnik-Brown, R., Porazinska, D.L., Weiss, S.J., Knight, R. (2013). Toward Effective Probiotics for Autism and Other Neurodevelopmental 
Disorders, Cell 155:1446-1448.

Gill, S.S. and Tuteja, N. (2010). Reactive oxygen species and antioxidant machinery in abiotic stress tolerance in crop plants. Plant Physiology and Biochemistry 48: 909-930.

Gong, L., Cao, W., Chi, H., Wang, J., Zhang, H., Liu, J., Sun, B. (2018). Whole cereal grains and potential health effects: Involvement of the gut microbiota. Food Research International 103: 84-102.

Granato, D., Branco, G.F., Nazzaro, F., Cruz, A.G. and Faria, J.A.F. (2010a). Functional Foods and Nondairy Probiotic Food Development: Trends, Concepts, and Products. Comprehensive Reviews in Food Science 9: 292-302.

Granato, D., Branco, G.F., Cruz, A.G., Faria, J. de A.F. and Shah, N.P. (2010b). Probiotic Dairy Products as Functional Foods. Comprehensive Reviews in Food Science 9: 455-470.

Hacquard, S., Garrido-Oter, R., González, A., Spaepen, S., Ackermann, G., Lebeis, S., McHardy, A.C., Dangl, J.L., Knight, R., Ley, R., and Schulze-Lefert, P. (2015). Microbiota and Host Nutrition across Plant and Animal Kingdoms. Cell Host Microbe 17(5): 603-616.

Halliwell, B. (2007). Dietary polyphenols: Good, bad, or indifferent for your health? Cardiovascular Research 73: 341-347.

Hettiarachchi, M., Liyanage, C., Wickremasinghe, R., Hilmers D.C. and Abrams S.A. (2006). Prevalence and severity of micronutrient deficiency: a cross-sectional study among adolescents in Sri Lanka. Asia Pacific Journal of Clinical Nutrition 15 (1):56-63.

Hong, H.A., Duc, L.H. and Cutting, S.M. (2005). The use of bacterial spore formers as probiotics. FEMS Microbiology Reviews 29: 813-835.

Hutkins, R.W., Krumbeck, J.A., Bindels, L.B., Cani, P.D. et al. (2016). Prebiotics: why definitions Matter. Current Opinion in Biotechnology 37: 1-7.

Iannitti, T. and Palmieri, B. (2010). Therapeutical use of probiotic formulations in clinical practice. Journal of Clinical Nutrition 29:701-725.

Jarchum, I. and Pamer, E.G. (2011). Regulation of innate and adaptive immunity by the commensal microbiota. Current Opinions in Immunology 23(3): 353-360.

Jayatissa, R., Gunathilaka, M.M., Fernando, D.N. (2012). National nutrition and Micronutrient survey. Part I: anaemia among children aged 6-59 months and nutritional status of children and adults, Ministry of Health, Sri Lanka/UNICEF Pp 63. https://www.unicef. org/srilanka/MNS_Report-28.02.2013.pdf Accessed November 30, 2017.

Jayawardena R., Byrne, N.M., Soares, M.J., Katulanda, P., Hills, A.P. (2012). Food consumption of Sri Lankan adults: an appraisal of serving characteristics. Public Health Nutrition: 16(4), 653-658.

Jones, M.L., Martoni, C.J. and Prakash, S. (2012). Cholesterol lowering and inhibition of sterol absorption by Lactobacillus reuteri NCIMB 30242: a randomized controlled trial. European Journal of Clinical Nutrition 66, 1234-1241.

Karlsson, C.L.J., Önnerfält J., Xu, J., Molin, G., Ahrné, S. and Thorngren-Jerneck, K. (2012). The Microbiota of the Gut in Preschool Children with Normal and Excessive Body Weight. Obesity 20(11): 2257-2261.

Karunaratne, A.M. (2007). Protein, energy, iron, zinc and vitamin A nutriture of 3-5 year group of children of low-socio-economic status in Kandy (Sri Lanka). Ph.D. thesis, Postgraduate Institute of Science, University of Peradeniya, Sri Lanka. Pp 238.

Karunaratne, A.M., Amerasinghe, P.H., Sadagopa Ramanujam, V.M., Sandstead, H.H. and Perera, P.A.J. (2008). Zinc, Iron and Phytic Acid levels of some popular foods consumed by rural children in Sri Lanka. Journal of Food Composition and Analysis 21: 481488.

Kavitake, D., Kandasamy, S., Devi, P.B., Shetty, P.H. (2018). Recent developments on encapsulation of lactic acid bacteria as potential starter culture in fermented foods - A review. Food Bioscience 21: 34-44.

Koplin, J.J., Peters, R.L., Allen, K.J. (2018). Prevention of Food Allergies. Immunology and Allergy Clinics of North America 38: (1) 1-11.

La Anh, N. (2015). Health-promoting microbes in traditional Vietnamese fermented foods: A review. Food Science and Human Wellness, 4: 147161.

Lozupone, C.A., Stombaugh, J.I., Gordon, J.I., Jansson, J.K. and Knight, R. (2012). Diversity, stability and resilience of the human gut microbiota Nature 489 :220230.

Marshall, E. and Mejia, D. (2011). Traditional fermented food and beverages for improved livelihood. Rural Infrastructure and Agro-Industries Division, Food and Agriculture Organization of the United Nations, Rome. http://www.fao.org/3/a-i2477e.pdf Accessed January 20, 2013.

Martins, E.M.F., Ramos, A.M., Vanzela, E.S.L., Stringheta, P.C., Pinto, C.L.O. and Martins J.M. (2013). Products of vegetable origin: A new alternative for the consumption of probiotic bacteria. Food Research International 51:764-770.

Marco, M.L., Heeney, D., Binda, S., Cifelli, C.J., Cotter, P.D., Foligne, B., Gänzle, M., Kort, R., Pasin, G., Pihlanto, A. Smid, E.J. and Hutkins, R. (2017). Health benefits of fermented foods: microbiota and beyond. Current Opinion in Biotechnology, 44: 94-102. Marinelli, L., Tenore, G. C. and Novellino, E. (2017). Probiotic species in the modulation of the anticancer immune response. In Seminars in Cancer Biology. 46: 182-190.

Martirosyan, D. M. and Singh, J. (2015). A new definition of functional food by FFC: what makes a new definition unique? Functional Foods in health and disease, 5(6): 209-223.

McFarland, L.V. (2015). From Yaks to Yoghurt: The History, Development, and Current Use of Probiotics. Clinical Infections and Disease 60(2): S85-S90.

Metchnikoff, E. (1908). The Prolongation of Life. Optimistic Studies. G. P. Putnam's Sons, London, England.

Morgan, X.C., Segata, N. and Huttenhower, C. (2013). Biodiversity and functional genomics in the human microbiome. Trends in Genetics 29(1): 51-58. 
Morris, V.J., Belshaw, N.J., Waldron, K.W. and Maxwell, E.G. (2013). The bioactivity of modified pectin fragments. Bioactive Carbohydrates and Dietary Fibre 1: 21-37.

Naidu, K.S.B., Adam, J.K. and Govender, P. (2012). The use of probiotics and safety concerns: A review. African Journal of Microbiological Research 6(41): 6871-6877.

Nakamura, Y.K. and Omaye, S.T. (2012). Metabolic diseases and pro- and prebiotics: Mechanistic insights. Nutrition and Metabolism 9:60. doi:10.1186/17437075-9-60 Accessed November 21, 2014.

Nakamura, F., Ishida, Y., Aihara, K., Sawada, D., Ashida, N., Sugawara, T., Aoki, Y., Takehara, I., Takano, K. and Fujiwara, S. (2016). Effect of fragmented Lactobacillus amylovorus $\mathrm{CP} 1563$ on lipid metabolism in overweight and mildly obese individuals: a randomized controlled trial. Microbial Ecology in Health and Disease, 27(1): 303-312.

Nowrouzian, F.L., Ali, A., Badiou, C., Dauwalder, O., Lina, G. and Josefsson, E. (2015). Impacts of enterotoxin gene cluster-encoded superantigens on local and systemic experimental Staphylococcus aureus infections. European Journal of Clinical Microbiology \& Infectious Diseases 34(7): 1443-1449.

Oh J., and Segre, J.A. (2012). Genomics: Resident risks. Nature 490: 44-46.

Panghal, A., Janghub, S., Virkara, K., Gata, Y., Kumara, V., Chhikaraa, N. (2018). Potential non-dairy probiotic products-A healthy approach. Food Bioscience 21: 8089.

Patra, J.K., Das, G., Paramithiotis, S. and Shin, H.S. (2016). Kimchi and other widely consumed traditional fermented foods of Korea: a review. Frontiers in Microbiology 7:1493. doi: 10.3389/fmicb.2016.01493 Accessed December 10, 2017.

Permpoonpattana, P., Hong, H.A., Khaneja, R. and Cutting, S.M. (2012). Evaluation of Bacillus subtilis strains as probiotics and their potential as a food ingredient. Beneficial Microbes 3(2): 127-135.

Phillips, G.O. (2013). Dietary fibre: A chemical category or a health ingredient? Bioactive Carbohydrates and Dietary Fibre 1(1): 3-9.

Qin, J., Li, Y., Cai, Z. et al. (2012). Metagenome-wide association study of gut microbiota in type 2 diabetes. Nature 490: 55-60.

Ray.M., Ghosh, K., Singh, S., Mondal, K.C. (2016). Folk to functional: An explorative overview of rice-based fermented foods and beverages in India. Journal of Ethnic Food 3: 5-18.

Rehman, A., Heinsen. F., Koenen, M.E., Venema, K., Knecht, H., Hellmig, S., Schreiber, S., Ott, S.J. (2012). Effects of probiotics and antibiotics on the intestinal homeostasis in a computer controlled model of the large intestine. BMC Microbiology 12(47) 10.1186/14712180-12-47 Accessed May 12, 2014.

Rizzo, L., Manaia, C., Merlin, C., Schwartz, T., Dagot, C., Michael, M.C.C.I. and Fatta-Kassinos, D. (2013). Urban wastewater treatment plants as hotspots for antibiotic resistant bacteria and genes spread into the environment: A review. Science of the Total
Environment 447: 345-360.

Roy, S. and Trinchieri, G. (2017). Microbiota: a key orchestrator of cancer therapy. Nature Reviews Cancer, 17(5): 271-285.

Ruan, Y., Sun, J., He, J., Chen, F., Chen, R. and Chen, H. (2015). Effect of probiotics on glycemic control: a systematic review and meta-analysis of randomized, controlled trials. PloS ONE doi: 10.1371/journal. pone.0132121 Accessed August 15, 2016.

Rouxinol-Dias, A. L., Pinto, A. R., Janeiro, C., Rodrigues, D., Moreira, M., Dias, J. and Pereira, P. (2016). Probiotics for the control of obesity-Its effect on weight change. Porto Biomedical Journal 1(1), 12-24.

Saad, N., Delattre, C., Urdaci, M., Schmitter, J.M. and Bressollier, P. (2013). An overview of the last advances in probiotic and prebiotic field. LWT-Food Science and Technology 50:1-16.

Sahlin, P. (1999). Fermentation as a Method of Food Processing Licentiate thesis, Lund Institute of Technology, Sweden. Pp 63.

Sang, L.X., Chang, B., Wang, B.Y., Liu, W.X. and Jiang, M., (2015). Live and heat-killed probiotic: effects on chronic experimental colitis induced by dextran sulfate sodium (DSS) in rats. International Journal of Clinical and Experimental Medicine, 8(11): 200-272.

Sawada, D., Sugawara, T., Ishida, U., Aihara, K., Aoki, Y., Takehara, Y., Takano, K. and Fujiwara, S. (2016). Effect of continuous ingestion of a beverage prepared with Lactobacillus gasseri CP2305 inactivated by heat treatment on the regulation of intestinal function, Food Research International 79: 33-39.

Seneviratne, M., Gunasinghe, W.K.R.N., GamaArachchige, N.S., Dissanayake, N.B.U. and Karunaratne, A. M. (2012). Mineral bioavailability in three locally consumed pulses processed using popular methods: interpreted using molar ratios with phytic acid. Ceylon Journal of Science (Biological Sciences) 41(1): 19-26.

Shahidi, F. (2004). Functional Foods: Their Role in Health Promotion and Disease Prevention. Journal of Food Science 69 (5): R146-R149.

Shalini, R., Abinaya, G., Saranya, P. and Antony, U. (2017). Growth of selected probiotic bacterial strains with fructans from Nendran banana and garlic. LWTFood Science and Technology, 83: 68-78.

Shinkai, S. Toba, M. Saito, T. et al. (2013). Immunoprotective effects of oral intake of heatKilled Lactobacillus pentosus strain b240 in elderly adults: a randomised, double-blind, placebo-controlled trial. British Journal of Nutrition 109, 1856-1865.

Shori, A.B., (2017). Microencapsulation Improves Probiotics Survival During Gastric Transit. HAYATI Journal of Biosciences 24: 1-5. http://dx.doi. org/10.1016/j.hjb.2016.12.008 Accessed December 10, 2017.

Singh, B., Mal, G. and Marotta, F. (2017). Designer Probiotics: Paving the Way to Living Therapeutics. Trends in Biotechnology. 35(8): 679-682. Sugawara T, Sawada D, Ishida Y, et al. (2016). Regulatory effect of paraprobiotic Lactobacillus gasseri CP2305 
on gut environment and function. Microbial Ecology in Health and Disease. 27:10.3402/mehd.v27.30259. doi:10.3402/mehd.v27.30259. Accessed December 10, 2017.

Sun-Waterhouse, D. (2011). The development of fruitbased functional foods targeting the health and wellness market: a review. International Journal of Food Science and Technology 46: 899-920.

Swain, M.R., Anandharaj, M., Ray, R.C. and Parveen, R. (2014). Fermented fruits and vegetables of Asia: a potential source of probiotics. Biotechnology Research International, Article ID 250424, 19 pages. Hindawi Publishing Corporation http://dx.doi. org/10.1155/2014/250424 Accessed November 30, 2015.

Tamang, J.P., (2015). Naturally fermented ethnic soybean foods of India. Journal of Ethnic Foods, 2(1): 8-17.

Taranu, I., Marin, D.E., Manda, G., Burghelea, M. and Tabuc, C. (2010). Potential of a lactobacilli probiotic to counteract the toxic effects of Fusarium mycotoxins. Proceedings of the Nutrition Society, Girona, Spain, 69: E283.

Taverniti, V. and Guglielmetti, S. (2011). The immunomodulatory properties of probiotic microorganisms beyond their viability (ghost probiotics: proposal of paraprobiotic concept). Genes \& Nutrition 6(3): 261-274.

The Human Microbiome Consortium (2012). Structure, function and diversity of the healthy human microbiome. Nature 486: 207-214.

Thomas, F., Hehemann, J.H., Rebuffet, E., Czjzek, M., Michel, G. (2011). Environmental and gut bacteroidetes: the food connection. Frontiers in Microbiology 2: 1-16.

Torres-Fuentes, C. Schellekens, H. and Cryan, J.F. (2017). The microbiota-gut-brain axis in obesity. Gastroenterology \& Hepatology The Lancet 2(10): 747-756.

Trebichavsky, I., Splichal. I., Rada, V. and Splichalova, A. (2010). Modulation of natural immunity in the gut by Escherichia coli strain Nissle 1917. Nutrition Reviews 68: 459-464.

Tremaroli, V. and Bäckhed, F. (2012). Functional interactions between the gut microbiota and host metabolism. Nature 489: 242-249.

Tripathi, M.K., Giri, S.K. (2014). Probiotic functional foods: Survival of probiotics during processing and storage. Journal of functional foods 9: 225-241.

Tsukumo, D.M., Carvalho, B.M., Carvalho-Filho, M.A., Saad, M.J.A. (2009). Translational research into gut microbiota: new horizons in obesity treatment. Arquivos Brasileiros de Endocrinologia \& Metabologia 53(2): 139-144.

Tudawe P.I., and Wikramanayake, T.W. (2000). Controlling anemia in Sri Lanka: Issues and options. Nutrition Coordination Division, Ministry of Plan Implementation and Parliamentary affairs, Sri Lanka USAID/OMNI. 1-82.

Ubeysekara, N.H., Jayathissa, R.,Wijesinghe C.J. (2015). Nutritional status and associated feeding practices among children aged 6-24 months in a selected community in Sri Lanka: a cross sectional study.
European Journal of Preventive Medicine. special issue: New Frontiers of Public Health From The Pearl of Indian Ocean, Sri Lanka. 3(2-1): 15-23.

Velasquez-Manoff, M. (2015). Gut Microbiome: The Peacekeepers. Nature 518: S3-S11.

Ventura, M., Turroni, F., Motherway, M.O., MacSharry, J. and van Sinderen, D. (2012). Host-microbe interactions that facilitate gut colonization by commensal bifidobacteria. Trends in Microbiology 20(10): 467476.

Vieira, C.P., Claudius, C.C. da Costa, B.R.C., et al., (2017). Lactococcus lactis ssp. cremoris MRS47, a potential probiotic strain isolated from kefir grains, increasescis-9, trans-11-CLA and PUFA contents in fermented milk. Journal of Functional Foods 31: 172178.

Viggiano, D., Ianiro, G., Vanella, G., Bibbò, S., Bruno, G., Simeone, G. and Mele, G. (2015). Gut barrier in health and disease: focus on childhood. European Review of Medical Pharmacology 19 (6): 1077-1085.

Whipps, J.M., Hand, P., Pink, D.A.C. and Bending, G.D. (2008). Human Pathogens and the Phyllosphere. Advances in Applied Microbiology 64: 183-221.

WHO (2013). Global strategy on diet, physical activity and health; Promoting fruit and vegetable consumption around the world. http:/www.who.int/ dietphysicalactivity/fruit/en/index $2 . \mathrm{html}$ Accessed October 21, 2015.

Willyard, C. (2011). Gut reaction: Microbes are under the spotlight in efforts to unravel and combat allergies. Nature 479: S5-S7.

Zhou, K. (2017). Strategies to promote abundance of Akkermansia muciniphila, an emerging probiotics in the gut, evidence from dietary intervention studies. Journal of Functional Foods 33: 194-201. 